\title{
Ultrathin Oxide Films by Atomic Layer Deposition on Graphene
}

\author{
Luda Wang, ${ }^{\dagger}$ Jonathan J. Travis, ${ }^{\ddagger}$ Andrew S. Cavanagh, ${ }^{\ddagger}$ Xinghui Liu, ${ }^{\dagger}$ Steven P. Koenig, ${ }^{\dagger}$ \\ Pinshane Y. Huang, ${ }^{\S}$ Steven M. George, ${ }^{\ddagger}$ and J. Scott Bunch*, ${ }^{*}$ \\ ${ }^{\dagger}$ Department of Mechanical Engineering and Department of Chemistry and Biochemistry, University of Colorado, Boulder, \\ Colorado 80309, United States \\ ${ }^{\S}$ School of Applied and Engineering Physics, Cornell University, Ithaca, New York 14853, United States
}

\section{Supporting Information}

ABSTRACT: In this paper, a method is presented to create and characterize mechanically robust, free-standing, ultrathin, oxide films with controlled, nanometer-scale thickness using atomic layer deposition (ALD) on graphene. Aluminum oxide films were deposited onto suspended graphene membranes using ALD. Subsequent etching of the graphene left pure aluminum oxide films only a few atoms in thickness. A pressurized blister test was used to determine that these ultrathin films have a Young's modulus of $154 \pm 13 \mathrm{GPa}$. This Young's modulus is comparable to much thicker alumina ALD films. This behavior indicates that these ultrathin twodimensional films have excellent mechanical integrity. The films are also impermeable to standard gases suggesting they are pinhole-free. These continuous ultrathin films are expected to enable new applications in fields such as thin film coatings, membranes, and flexible electronics.

KEYWORDS: Atomic layer deposition, graphene, nanomechanics, thin films

$\mathrm{T}$ wo-dimensional (2D) materials are promising nanomechanical structures. ${ }^{1,2}$ Graphene, the best known and studied of this class of materials, boasts a high Young's modulus, intrinsic strength, gas impermeability, and excellent thermal and electrical conductivity. ${ }^{3-8}$ There are numerous applications where flexible ultrathin insulating or oxide films are needed with comparable mechanical properties. The integration of graphene with other $2 \mathrm{D}$ or quasi-2D materials may also lead to new functional properties for the composite materials. ${ }^{9-13}$ Currently, the range of ultrathin materials is severely limited by the materials and length-scales that are accessible through thin film fabrication.

Mechanical and chemical exfoliation, as well as growth techniques such as chemical vapor deposition, can produce just a handful of ultrathin layered materials. ${ }^{1,14-17}$ As traditional materials approach $\sim 1 \mathrm{~nm}$ film thicknesses, fabrication of freely suspended films is difficult due to stresses or significant voids in the films that destroy the mechanical integrity of the film. To overcome these problems, we use suspended graphene membranes as sacrificial supports to grow high quality ALD films and then remove the graphene to leave the ALD thin film. These experiments demonstrate that ALD on graphene offers a route to create free-standing, ultrathin, quasi-2D structures with atomically controlled thickness and mechanical properties comparable to their bulk counterparts. ${ }^{18-21}$

Atomic layer deposition films are fabricated using a combination of deposition and etching using a suspended graphene support. The graphene provides an atomically smooth growth surface that can easily be etched away. Graphene is mechanically exfoliated over predefined wells as illustrated in Figure 1a. The graphene is then exposed to a trimethylaluminum (TMA) and $\mathrm{NO}_{2}$ treatment that forms an adhesion layer for ALD nucleation. ${ }^{22-24}$ Aluminum oxide ALD is subsequently grown using TMA $/ \mathrm{H}_{2} \mathrm{O}$ doses ${ }^{25,26}$ (see Supporting Information). An example of such a graphene/ALD composite film after seven cycles of alumina ALD is shown in Figure 1b. Highresolution cross-sectional transmission electron microscopy on such a graphene sample with a TMA/ $\mathrm{NO}_{2}$ adhesion layer followed by five cycles of TMA $/ \mathrm{H}_{2} \mathrm{O}$ shows the ALD film to be amorphous and $2.8 \pm 0.3 \mathrm{~nm}$ thick (see Supporting Information). We then use oxidative etching of the underlying graphene support to leave only the thin alumina ALD film suspended over the predefined well as displayed in Figure 1c. Oxidative etching is carried out in a $1 \mathrm{in}$. diameter tube furnace at $600{ }^{\circ} \mathrm{C}$ with an $\mathrm{O}_{2}$ gas flow of $\sim 20-40 \mathrm{ccm}$ for $\sim 10 \mathrm{~h}$. This is sufficient to completely etch away the graphene. After the graphene is etched away, the film is no longer visible in the optical microscope, and Raman spectroscopy that shows no signs of a substantial D, G, and $2 \mathrm{D}$ peak in the etched samples is used to confirm the absence of graphene (see Supporting Information).

Received: April 20, 2012

Revised: June 7, 2012

Published: June 20, 2012 

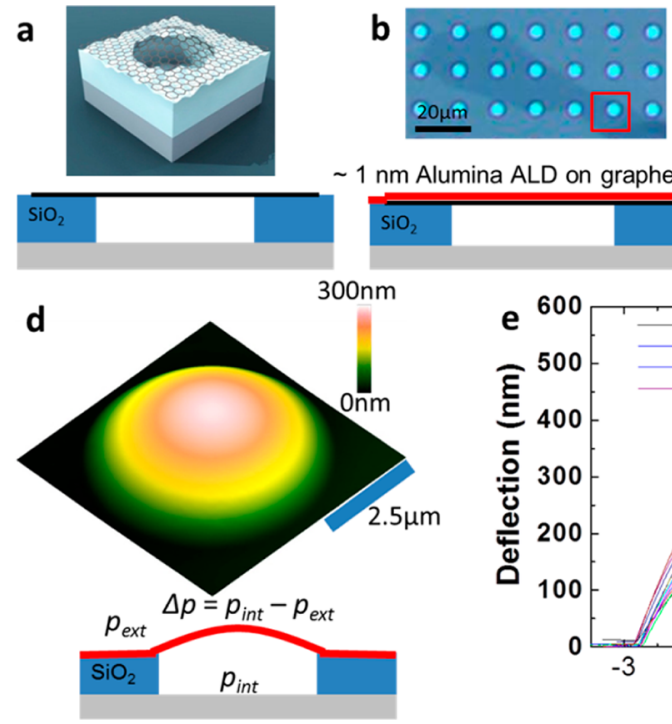

C

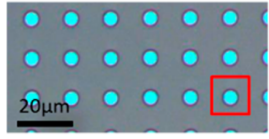

m Alumina ALD on graphene $\sim 1 \mathrm{~nm}$ Alumina ALD
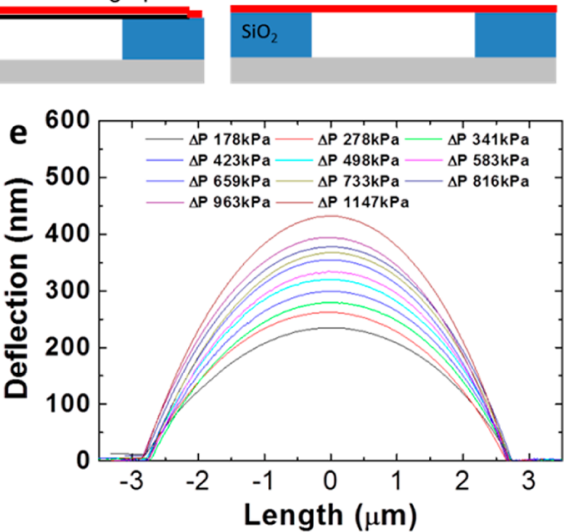

Figure 1. (a) Schematic of a graphene membrane before atomic layer deposition (ALD). (b) (upper) Optical image of an exfoliated graphene flake with seven cycles of alumina ALD. (lower) Side view schematic of this graphene/ALD composite. (c) (upper) Optical image of a pure alumina film after graphene is etched away. (lower) Side view schematic of this pure ALD film. (d) (upper) Atomic force microscope image of a pressurized seven cycle pure alumina ALD film with $\Delta p=278 \mathrm{kPa}$. This film corresponds to the film boxed in red in (b) and (c). (lower) Side view schematic of a pressurized ALD film. (e) Deflection vs position through the center of the film in (d) at different $\Delta p$.
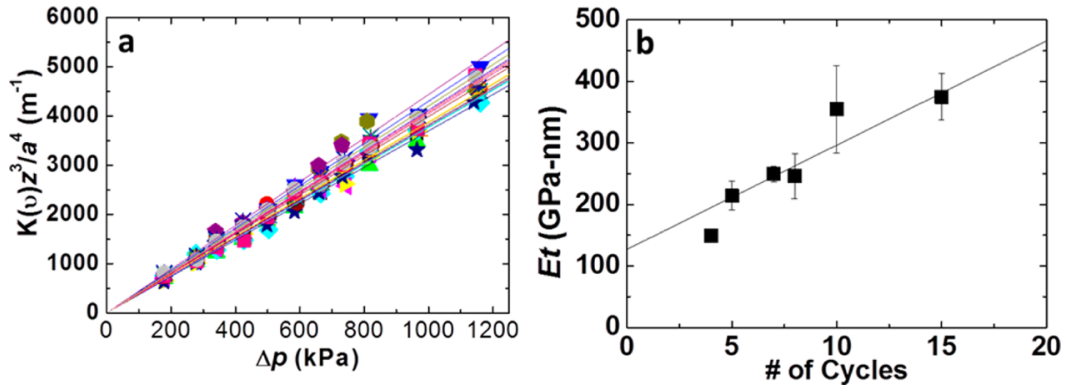

Figure 2. (a) $K(v) z^{3} / a^{4}$ versus $\Delta p$ for 18 pure ALD films with 7 cycles of alumina ALD. Colored lines are best fits to each sample. The average and standard deviation of all the slopes corresponds to $E t=250 \pm 12 \mathrm{GPa}-\mathrm{nm}$. (b) Et vs number of cycles for all the pure ALD films measured. The standard deviation is shown as error bars. The solid line is a best fit to the data and corresponds to $E t_{\text {cycle }}=16.9 \pm 1.4 \mathrm{GPa}-\mathrm{nm}$ with an intercept of $E_{0} t_{0}=127.1 \pm 13.1 \mathrm{GPa}-\mathrm{nm}$. This corresponds to $E_{\mathrm{ALD} \mathrm{Al}} \mathrm{O}_{3}=154 \pm 13 \mathrm{GPa}$ assuming a thickness gain per cycle of $t_{\text {cycle }}=0.11 \mathrm{~nm}$.

A pressure difference is applied to the film using a previously reported method where slow diffusion through the $\mathrm{SiO}_{2}$ substrate over pressurizes the film sealed microchamber. ${ }^{5,27}$ An atomic force microscope (AFM) image of such an over pressurized suspended film in Figure 1c is shown in Figure 1d. The ALD film is bulged upward with a maximum deflection through the center of the film, $\delta=261 \mathrm{~nm}$, and a radius, $a=$ $2.76 \mu \mathrm{m}$, consistent with the radius of the predefined well. At increasing $\Delta p$, the film stretches further as $\delta$ increases as characterized in Figure 1e. During AFM imaging, the bulge is stable suggesting a constant pressure difference and no significant leak rate of gas out of the microchamber, similar to previous results on graphene membranes. ${ }^{5}$ This behavior implies that the aluminum oxide films are pinhole-free and impermeable to the nitrogen gas used for pressurization.

The deformation of the film follows ${ }^{27,28}$

$$
\Delta p=K(v) \frac{\delta^{3}}{a^{4}} E t
$$

where $E$ is Young's modulus, $t$ is the thickness of the film, and $K(v)$ is a constant that depends on the Poisson's ratio. For the case of aluminum oxide, $K(v=0.24)=3.35$. Figure 2 a shows
$K(v)\left(\delta^{3} / a^{4}\right)$ versus $\Delta p$ for 18 pure alumina ALD films (graphene etched away) fabricated on an exfoliated graphene flake using seven cycles of alumina ALD. The behavior of each film follows a line as expected from eq 1. The average and standard deviation of the slope of these lines gives $E t=250 \pm$ 12 GPa-nm.

A similar measurement was performed for a number of different films formed using 4-15 cycles. The plot of Et versus number of ALD cycles is shown in Figure 2b. A best fit line of the data gives a slope of $E t_{\text {cycle }}=16.9 \pm 1.4 \mathrm{GPa}-\mathrm{nm}$ with an intercept of $E_{0} t_{0}=127.1 \pm 13.1 \mathrm{GPa}-\mathrm{nm}$. This nonzero intercept likely arises from the $E t$ value of the functionalization layer. This slope corresponds to $E_{\mathrm{ALD} \mathrm{Al}_{2} \mathrm{O}_{3}}=154 \pm 13 \mathrm{GPa}$ assuming an ALD growth rate of $0.11 \mathrm{~nm} /$ cycle. $^{25,29}$ This Young's modulus is comparable to previous measurements on much thicker (tens to hundreds of nm) alumina ALD films that have Young's moduli of $168-220 \mathrm{GPa}^{30-32}$ Because the films are freely suspended, a mechanical support does not influence the mechanical properties of the ALD thin films. The high Young's modulus is remarkable considering our samples are 23 orders of magnitude thinner than previously measured ALD films. 

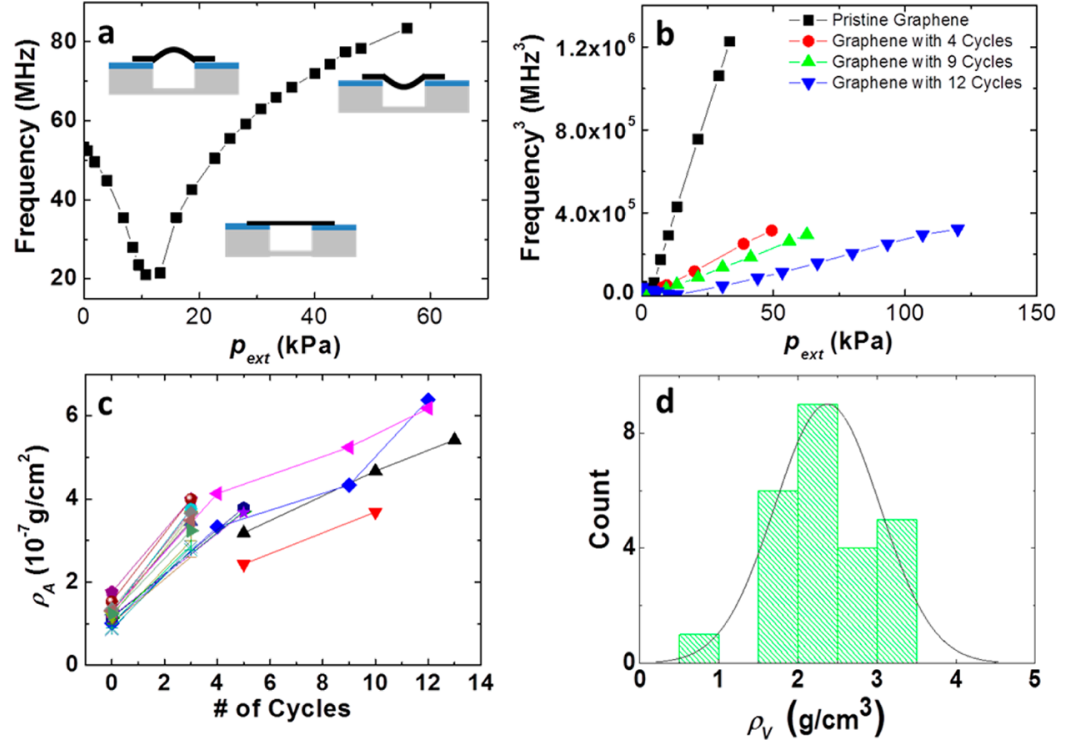

Figure 3. (a) Mechanical resonant frequency vs $p_{\text {ext }}$ for a graphene/ALD composite film with five cycles of alumina ALD. (insets) Schematic of the film at different $\Delta p$. (b) Frequency ${ }^{3}$ vs $p_{\text {ext }}$ for a single graphene/ALD composite film with 0, 4, 9, 12 cycles of alumina ALD. (c) Areal mass density $\rho_{\mathrm{A}}$ vs number of cycles for all the graphene/ALD composites measured. (d) Histogram of volume mass density $\rho_{\mathrm{V}}$ for the alumina ALD films. The black line is a Gaussian fit to the data.
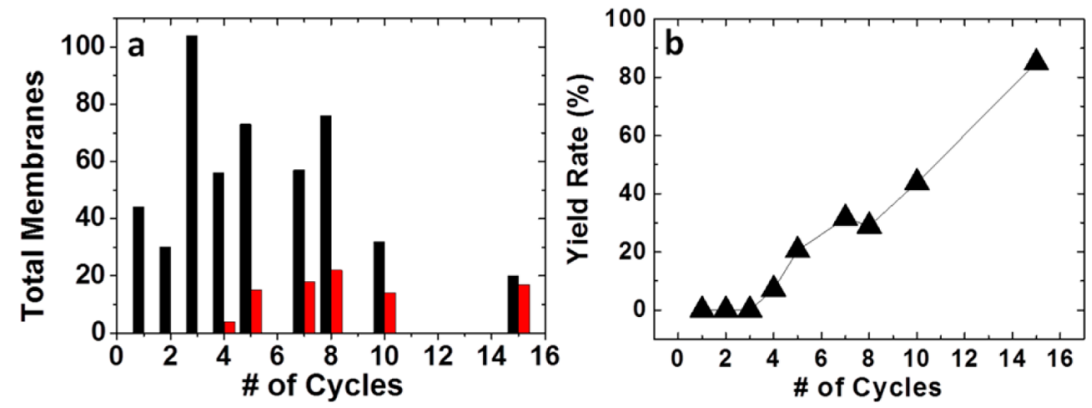

Figure 4. (a) (black) Number of all pure ALD films fabricated in this study vs number of ALD coating cycles. (red) Number of pure ALD films that hold $\mathrm{N}_{2}$ gas from that sample batch (b) Percentage yield vs number of cycles for all the pure ALD films fabricated.

The pressure induced-strain in the film can be used to tune the mechanical resonance frequency of the suspended films. Figure $3 \mathrm{a}$ demonstrates this behavior for a graphene/ALD composite film fabricated using five cycles of alumina ALD. The mechanical resonance is actuated and detected optically as previously reported. ${ }^{3,5}$ We were unable to measure a resonance frequency for the pure alumina ALD films presumably due to the lack of optical reflectivity from these samples. The frequency first decreases and then increases as the film transitions from a bulged upward to a bulged downward state.

At sufficiently large pressures far from the minimum frequency, the frequency scales as $f^{3} \alpha \Delta p$. The slope shows a dramatic decrease in frequency with the addition of alumina ALD cycles as shown in Figure $3 \mathrm{~b}$. This behavior can be explained by the pressure-induced changes in the tension in a stretched circular film according to

$$
f^{3}=7 \times 10^{-3} \sqrt{\frac{K(v) E t}{a^{4} \rho_{\mathrm{A}}^{3}}} \Delta p
$$

where $\rho_{\mathrm{A}}$ is the mass per unit area. From the slope of the lines in Figure $3 \mathrm{~b}$ and using $\mathrm{K}(v) E t$ determined by a pressurized blister test on the composite $\mathrm{ALD} /$ graphene film (see Supporting Information), we can determine $\rho_{\mathrm{A}}$ of each suspended film before and after each ALD process. All samples showed an increase in $\rho_{\mathrm{A}}$ with the number of alumina ALD cycles as displayed in Figure 3c. The first three cycles showed a larger increase in $\rho_{\mathrm{A}}$ that may be related to the initial nucleation of alumina ALD. The finite $\rho_{\mathrm{A}}$ before any ALD cycles is attributed to the additional mass from the adhesion layer.

Using the measured $\rho_{A}$, we can estimate the volume density of the ALD films, $\rho_{\mathrm{V}}$, and the areal mass density of the adhesion layer, $\rho_{\mathrm{A} \_ \text {ad }}$. Because all the samples have an adhesion layer with an unknown $\rho_{\mathrm{A} \text { ad }}$, we first determine $\rho_{\mathrm{V}}$ from the slope of the lines in Figure $\overline{3} \mathrm{c}$ for coatings after the nucleation treatment. This determination yields $\rho_{\mathrm{V}}=2.3 \pm 0.4 \mathrm{~g} / \mathrm{cm}^{3}$ assuming an ALD growth rate of $0.11 \mathrm{~nm} /$ cycle. $^{25}$ (The anomalously large value at 12 cycles shown in blue was not used in calculating this average and standard deviation.) We then deduce $\rho_{\mathrm{A} \text { ad }}$ from the measured $\rho_{\mathrm{A}}$ using $\rho_{\mathrm{A} \_ \text {ad }}=\left(\rho_{\mathrm{A}}-\rho_{\mathrm{V}}\right) N$, where $\bar{N}$ is the number of alumina ALD cycles. This derivation yields an average value and standard deviation of $\rho_{\mathrm{A} \text { ad }}=1.4 \pm 0.3 \times$ $10^{-7} \mathrm{~g} / \mathrm{cm}^{2}$. We can then determine $\rho_{\mathrm{V}}$ for every ALD film in Figure 3c. This procedure yields $\rho_{\mathrm{V}}=2.4 \pm 0.7 \mathrm{~g} / \mathrm{cm}^{3}$ as shown in Figure $3 \mathrm{~d}$. This density is comparable within experimental error to previous densities measured on thicker alumina ALD thin films of $3.0 \mathrm{~g} / \mathrm{cm}^{3}$. 
None of the 178 samples fabricated with less than 4 alumina ALD cycles were impermeable to $\mathrm{N}_{2}$ gas after removal of the graphene as shown in Figure 4a. However, the yield of impermeable films increased with number of ALD cycles and reached $85 \%$ for 15 ALD cycles as displayed in Figure $4 \mathrm{~b}$. This behavior indicates that increasing the number of ALD cycles reduces pinholes or gas diffusion through the film.

For freely suspended films formed using only five cycles of the TMA $/ \mathrm{NO}_{2}$ nucleation treatment, AFM images of the films do not show voids (see Supporting Information). This corroborates our measurements of a contribution from the adhesion layer to $E$ and $\rho_{\mathrm{A} \text { funct }}$ in Figures $2 \mathrm{~b}$ and $3 \mathrm{c}$. Future work will examine the dependence of the adhesion layer and its role in nucleating continuous pinhole-free ALD alumina thin film growth on graphene.

In conclusion, a new class of ultrathin films has been created based on aluminum oxide ALD on graphene. These films are mechanically robust, pinhole-free, and have approximately nanometer thicknesses while still maintaining a Young's modulus comparable to their much thicker counterparts. The manufacturability, thickness control, and versatility of the ALD process means that materials and processing can be tailored to suit many applications where traditional silicon or graphenebased thin film mechanical devices fail to offer the needed functionality. ${ }^{34,35}$ Furthermore, these films can be integrated with graphene or other nanomechanical structures to create multifunctional quasi-2D electromechanical structures.

\section{ASSOCIATED CONTENT}

\section{(5 Supporting Information}

Experimental methods, TEM imaging of a graphene/ALD composite, Raman spectrum verifying the etching of graphene, elastic constants of pure ALD films, initial tension in graphene and graphene/ALD composite films, and pure ALD films from the nucleation treatment. This material is available free of charge via the Internet at http://pubs.acs.org.

\section{AUTHOR INFORMATION}

\section{Corresponding Author}

*E-mail: jbunch@colorado.edu.

\section{Notes}

The authors declare no competing financial interest.

\section{ACKNOWLEDGMENTS}

We thank Darren McSweeney and Michael Tanksalvala for help with the resonance measurements, Rishi Raj for use of the Raman microscope, and Narasimha Boddetti, Jianliang Xiao, Martin L. Dunn, Victor Bright, Todd Murray, David Muller, Paul McEuen, and Yifu Ding for useful discussions. This work was supported by NSF Grants 0900832(CMMI: Graphene Nanomechanics: The Role of van der Waals Forces), 1054406(CMMI: CAREER: Atomic Scale Defect Engineering in Graphene Membranes), the DARPA Center on Nanoscale Science and Technology for Integrated Micro/Nano-Electromechanical Transducers (iMINT), the National Science Foundation (NSF) Industry/University Cooperative Research Center for Membrane Science, Engineering and Technology (MAST), and in part by the NNIN and the National Science Foundation under Grant ECS-0335765. Electron microscopy facilities were provided by the NSF through the Cornell Center for Materials Research (NSF DMR-1120296).

\section{REFERENCES}

(1) Novoselov, K. S.; Jiang, D.; Schedin, F.; Booth, T. J.; Khotkevich, V. V.; Morozov, S. V.; Geim, A. K. Proc. Natl. Acad. Sci. U.S.A. 2005, 102, 10451-10453.

(2) Geim, A. K. Science 2009, 324, 1530-1534.

(3) Bunch, J. S.; van der Zande, A. M.; Verbridge, S. S.; Frank, I. W.; Tanenbaum, D. M.; Parpia, J. M.; Craighead, H. G.; McEuen, P. L. Science 2007, 315, 490-493.

(4) Lee, C.; Wei, X.; Kysar, J. W.; Hone, J. Science 2008, 321, 385388.

(5) Bunch, J. S.; Verbridge, S. S.; Alden, J. S.; Zande, A. M. V. D.; Parpia, J. M.; Craighead, H. G.; McEuen, P. L. Nano Lett. 2008, 8, 2458-2462.

(6) Novoselov, K. S.; Geim, A. K.; Morozov, S. V.; Jiang, D.; Katsnelson, M. I.; Grigorieva, I. V.; Dubonos, S. V.; Firsov, A. A. Nature 2005, 438, 197-200.

(7) Zhang, Y. B.; Tan, Y. W.; Stormer, H. L.; Kim, P. Nature 2005, 438, 201-204.

(8) Balandin, A. A.; Ghosh, S.; Bao, W.; Calizo, I.; Teweldebrhan, D.; Miao, F.; Lau, C. N. Nano Lett. 2008, 8, 902-907.

(9) Dean, C. R.; Young, A. F.; Meric, I.; Lee, C.; Wang, L.; Sorgenfrei, S.; Watanabe, K.; Taniguchi, T.; Kim, P.; Shepard, K. L.; Hone, J. Nat Nanotechnol. 2010, 5, 722-726.

(10) Williams, J. R.; DiCarlo, L.; Marcus, C. M. Science 2007, 317, 638-641.

(11) Rogers, J. A.; Lagally, M. G.; Nuzzo, R. G. Nature 2011, 477, $45-53$.

(12) Jen, S.-H.; Bertrand, J. A.; George, S. M. J. Appl. Phys. 2011, 109, 84305-84311.

(13) Huang, P. Y.; Kurasch, S.; Srivastava, A.; Skakalova, V.; Kotakoski, J.; Krasheninnikov, A. V.; Hovden, R.; Mao, Q.; Meyer, J. C.; Smet, J.; Muller, D. A.; Kaiser, U. Nano Lett. 2012, 12, 1081-1086.

(14) Coleman, J. N.; Lotya, M.; O’Neill, A.; Bergin, S. D.; King, P. J.; Khan, U.; Young, K.; Gaucher, A.; De, S.; Smith, R. J.; Shvets, I. V.; Arora, S. K.; Stanton, G.; Kim, H.-Y.; Lee, K.; Kim, G. T.; Duesberg, G. S.; Hallam, T.; Boland, J. J.; Wang, J. J.; Donegan, J. F.; Grunlan, J. C.; Moriarty, G.; Shmeliov, A.; Nicholls, R. J.; Perkins, J. M.; Grieveson, E. M.; Theuwissen, K.; McComb, D. W.; Nellist, P. D.; Nicolosi, V. Science 2011, 331, 568-571.

(15) Li, X.; Cai, W.; An, J.; Kim, S.; Nah, J.; Yang, D.; Piner, R.; Velamakanni, A.; Jung, I.; Tutuc, E.; Banerjee, S. K.; Colombo, L.; Ruoff, R. S. Science 2009, 324, 1312-1314.

(16) Zhan, Y.; Liu, Z.; Najmaei, S.; Ajayan, P. M.; Lou, J. Small 2012, $8,966--971$.

(17) Suk, J. W.; Murali, S.; An, J.; Ruoff, R. S. Carbon 2012, 50, 2220-2225.

(18) Leskelä, M.; Ritala, M. Thin Solid Films 2002, 409, 138-146.

(19) Leskelä, M.; Ritala, M. Angew. Chem., Int. Ed. 2003, 42, 55485554.

(20) Elam, J. W.; Groner, M. D.; George, S. M. Rev. Sci. Instrum. 2002, 73, 2981-2987.

(21) Ritala, M.; Leskelä, M.; Dekker, J.-P.; Mutsaers, C.; Soininen, P. J.; Skarp, J. Chem. Vap. Deposition 1999, 5, 7-9.

(22) Cavanagh, A. S.; Wilson, C. a; Weimer, A. W.; George, S. M. Nanotechnology 2009, 20, 255602.

(23) Farmer, D. B.; Gordon, R. G. Nano Lett. 2006, 6, 699-703.

(24) Wang, X.; Tabakman, S. M.; Dai, H. J. Am. Chem. Soc. 2008, $130,8152-8153$.

(25) Ott, A. W.; Klaus, J. W.; Johnson, J. M.; George, S. M. Thin Solid Films 1997, 292, 135-144.

(26) Dillon, A. C.; Ott, A. W.; Way, J. D.; George, S. M. Surf. Sci. 1995, 322, 230-242.

(27) Koenig, S. P.; Boddeti, N. G.; Dunn, M. L.; Bunch, J. S. Nat. Nanotechnol. 2011, 6, 543-546.

(28) Hencky, H. Z. Math. Phys. 1915, 63, 311-317.

(29) Ott, A. W.; McCarley, K. C.; Klaus, J. W.; Way, J. D.; George, S. M. Appl. Surf. Sci. 1996, 107, 128-136. 
(30) Tripp, M. K.; Stampfer, C.; Miller, D. C.; Helbling, T.; Herrmann, C. F.; Hierold, C.; Gall, K.; George, S. M.; Bright, V. M. Sens. Actuators A: Physical 2006, 130-131, 419-429.

(31) Tapily, K.; Jakes, J. E.; Stone, D. S.; Shrestha, P.; Gu, D.; Baumgart, H.; Elmustafa, A. A. Journal of The Electrochemical Society 2008, 155, H545-H551.

(32) Miller, D. C.; Foster, R. R.; Jen, S.-H.; Bertrand, J. A.; Cunningham, S. J.; Morris, A. S.; Lee, Y.-C.; George, S. M.; Dunn, M. L. Sensors and Actuators, A 2010, 164, 58-67.

(33) Groner, M. D.; Fabreguette, F. H.; Elam, J. W.; George, S. M. Chem. Mater. 2004, 16, 639-645.

(34) Davidson, B. D.; Seghete, D.; George, S. M.; Bright, V. M. Sens. Actuators, A 2011, 166, 269-276.

(35) Yoneoka, S.; Lee, J.; Liger, M.; Yama, G.; Kodama, T.; Gunji, M.; Provine, J.; Howe, R. T.; Goodson, K. E.; Kenny, T. W. Nano Lett. 2012, 12, 683-6. 